\title{
BANACH SPACES WHICH ALWAYS CONTAIN SUPREMUM-ATTAINING ELEMENTS
}

\author{
PETER D. MORRIS
}

\begin{abstract}
It is proved that if a weakly compactly generated Banach space $\boldsymbol{X}$ has the property that, for every closed, bounded convex subset $K$ of $X^{*}$, there exists a nonzero element of $X$ which attains its supremum on $K$, then $X$ contains no copy of $l^{1}$.
\end{abstract}

Let $X$ be a Banach space and let $A$ be a bounded subset of its dual. Define

$$
S A(A)=\{x \in X \backslash\{0\}: \hat{x} \text { attains its supremum on } A\} .
$$

Here $\hat{x}$ denotes the image of $x$ under the natural embedding of $X$ in $X^{* *}$. Also define

$$
\operatorname{SEX}(A)=\{x \in X: \hat{x} \text { strongly exposes } A\} .
$$

Here, a functional $f$ strongly exposes a set $B$ if the diameter of the slice

$$
S(B, f, \alpha)=\{b \in B: f(b) \geqslant \alpha\}
$$

approaches 0 as $\alpha$ approaches $\max f(B)$ from below.

In case $A \subset X^{*}$ is norm-closed we have $S E X(A) \subset S A(A)$. This inclusion is usually proper.

The present work was motivated by the following results.

THEOREM A. The dual space $X^{*}$ has the Radon-Nikodym property if and only if $\operatorname{SEX}(A) \neq 0$ for all norm-closed, bounded, convex subsets, $A$ of $X^{*}$.

THEOREM B. The dual space $X^{*}$ has the Radon-Nikodym property if and only if $S A(A)$ is of 2 nd category for all norm-closed, bounded, convex subsets $A$ of $X^{*}$.

The reader should refer to [1] or [2] for information on the Radon-Nikodým property (including its definition). Theorem A follows from a result of Namioka and Phelps [4] combined with one of Stegall [7]. Indeed, their results imply that, if $X^{*}$ has the Radon-Nikodým property and $A$ is a norm-closed, convex subset, then $S E X(A)$ is a dense $G_{\delta}$ subset of $X$. This fact also proves half of Theorem B. The other half is a very easy modification of an argument due to Bourgain (see [1], [6]).

Theorems A and B, together with a spirit of optimism, led to

Conjecture. If $S A(A) \neq \varnothing$ for all norm-closed, bounded, convex subsets $A$ of $X^{*}$, then $X^{*}$ has the Radon-Nikodým property.

Let us refer to the property of $X$ which is conjectured to imply the $R N P$ in $X^{*}$ as Property $S A^{*}$. Our result is weaker than the one conjectured. We were forced to

Received by the editors April 15, 1980 and, in revised form, December 29, 1980.

1980 Mathematics Subject Classification. Primary 46B22; Secondary 46B10. 
assume that $X$ is weakly compactly generated. This allows a reduction to the separable case. The desired conclusion is that every separable subspace of $X$ has separable dual (that this implies that $X^{*}$ has the $R N P$ is due to Uhl [8] generalizing Dunford and Pettis [3]). We obtain the weaker conclusion that $l^{1}$ does not embed in $X$.

A lemma is needed.

LEMMA. $C[0,1]$ does not have property $S A^{*}$.

Proof. Let $\left(x_{n}\right)$ be a dense sequence in $[0,1]$. For each $n=1,2, \ldots$, let $\mu_{n}$ be the point mass at $x_{n}$ with total mass $n /(n+1)$. Let

$$
A=\overline{\operatorname{co}}\left(\left(\mu_{n}\right) \cup\left(-\mu_{n}\right)\right) \subseteq C([0,1])^{*} \text {. }
$$

It is easy to see that

$$
\sup \{\langle\mu, f\rangle: \mu \in A\}=\|f\|,
$$

for any $f \in C([0,1])$. Now we show that $\mu \in A$ then $\mu$ can be expressed as

$$
\mu=\sum_{n=1}^{\infty} a_{n} \mu_{n},
$$

where $\Sigma_{1}^{\infty}\left|a_{n}\right| \leqslant 1$. To prove this, let $T$ be the map from $l^{1}$ into $C([0,1])^{*}$ which takes $\left(a_{n}\right) \in l^{1}$ to $\Sigma_{1}^{\infty} a_{n} \mu_{n} \in C([0,1])^{*}$. Then $T$ is clearly an isomorphism. Since $A$ is obviously the image, under $T$, of the unit ball of $l^{1},(2)$ holds.

To finish the proof of the Lemma, we will show that no nonzero $f$ in $C([0,1])$ attains its supremum on $A$. Suppose the contrary. Then, using (1), there exists $f$ in $C([0,1])$ and $\mu \in A$ such that

$$
\langle\mu, f\rangle=\|f\|>0 .
$$

Expressing $\mu$ as in (2), we have

$$
\begin{aligned}
\|f\| & =\langle\mu, f\rangle=\sum_{1}^{\infty} a_{n}\left\langle\mu_{n}, f\right\rangle\left\langle\sum_{1}^{\infty}\left|a_{n} \|\left\langle\mu_{n}, f\right\rangle\right|\right. \\
& <\sum_{1}^{\infty}\left|a_{n}\right|\|f\| \leqslant\|f\| .
\end{aligned}
$$

This contradiction completes the proof of the Lemma.

THEOREM. Let $X$ be a weakly compactly generated Banach space with property $S A^{*}$. Then $l^{1}$ does not embed in $X$.

Proof. We first observe that every quotient of a space with property $S A^{*}$ also has this property. For, suppose $Q$ is a bounded linear operator from a Banach space $Y$ onto a Banach space $Z$. Suppose $K$ is a closed, bounded, convex set in $Z^{*}$ with $S A(K)=\varnothing$. A moments's reflection shows that $S A\left(Q^{*} K\right)=\varnothing$ and so $Y$ fails to have $S A^{*}$.

Now suppose that $l^{1}$ embeds in $X$. Since $X$ is weakly compactly generated there is a separable subspace $Y$ of $X$ which contains a copy of $l^{1}$ and which is complemented in $X$. Then $Y$ also has property $S A^{*}$. But it follows from a result of 
Pelczynski [5] that a separable space containing $l^{1}$ has $C[0,1]$ as a quotient. Hence $C[0,1]$ has $S A^{*}$ and we have arrived at a contradiction. This completes the proof.

We are grateful to J. Diestel, S. Fitzpatrick, and J. Rainwater for valuable discussions on the subject of this paper. In particular, it is probable that Fitzpatrick was the first to make the conjecture. We thank the referee for suggestions which led to great improvements in this paper.

\section{REFERENCES}

1. R. Bourgin, Notes on the Radon-Nikodym property (to appear).

2. J. Diestel, Geometry of Banach spaces, Lecture Notes in Math., vol. 485, Springer-Verlag, New York and Berlin, 1975.

3. N. Dunford and B. J. Pettis, Linear transformations on summable functions, Trans. Amer. Math. Soc. 47 (1940), 323-392.

4. I. Namioka and R. R. Phelps, Banach spaces which are Asplund spaces, Duke Math. J. 42 (1975), 735-750.

5. A. Pelczynski, On C(S)-subspaces of separable Banach spaces, Studia Math. 31 (1968), 513-522.

6. J. Rainwater, Univ. of Washington Seminar Notes, Fall 1976.

7. C. Stegall, The duality between Asplund spaces and spaces with the Radon-Nikodym property, Israel J. Math. 29 (1978), 408-412.

8. J. J. Uhl, A note on the Radon-Nikodym property for Banach spaces, Rev. Roumaine Math. Pures Appl. 17 (1972), 113-115.

Department of Mathematics, The Pennsylvania State University, University Park, PennsylVANIA 16802 